\title{
MOMENTS OF NONIDENTICAL ORDER STATISTICS FROM BURR XII DISTRIBUTION WITH GAMMA AND NORMAL OUTLIERS
}

\author{
Jamjoom, A.A. and Z.A. Al-Saiary \\ Department of Statistics, Girls College of Science-King, Abdul-Aziz University, Jeddah, Saudi Arabia
}

Received 2011-10-19, Revised 2013-01-09; Accepted 2013-04-12

\begin{abstract}
There are some distributions with no simple closed form for distribution functions such as the Normal and Gamma distributions. This will be the problem if we want to find moments of nonidentical order statistics in the presence of Gamma and Normal outliers observations. We used the idea of approximating Normal and Gamma distributions with Burr type XII distribution. We get single moments for order statistics from sample of independent nonidentically distributed Burr XII random variables that contains p-outlier from Normal or Gamma distributions. Approximating these distributions with Burr XII distribution and then we compared the results by previous method.
\end{abstract}

Keywords: Approximation, Nonidentical Order Statistics, Burr XII Distribution, Normal Distribution, PERMINANTS, Gamma Distribution

\section{INTRODUCTION}

There are no simple closed form exists for the normal distribution function and the gamma distribution function so that approximations to $\mathrm{G}(\mathrm{x})$ must be used to find moments of the rth identical order statistic. An approach of obtaining close approximation to the normal distribution was presented by Burr (1942). Burr (1967; 1973), Burr recalculated the values of shape parameters for Burr XII distribution. These values give a closer approximation to the normal distribution. Another approach based on approximating gamma distribution with the Burr family distribution has been presented by Tadikamalla and Ramberg (1975) and Wheeler (1975), who approximate Gamma with two parameters with Burr with two parameters. Tadikamalla (1977) used the generalized four parameter B-distribution to approximate gamma distribution and put a table for selected integral values of shape parameter and other parameters which Burr XII distribution function approximate to the exact Gamma distribution function. Corresponding Author: Jamjoom, A.A., Department of Statistics, Girls College of Science- King Abdul-Aziz University, Jeddah, Saudi Arabia

In this chapter we used the idea of approximating Normal and Gamma distributions with Burr XII to get single moments for order statistics from sample of independent nonidentically distributed Burr XII random variables that contains p-outlier from normal or Gamma distributions. approximating these distributions with Burr XII distribution and then we compared the results by those using the Barakat and Abdelkader (2004).

Barnett and Lewis (1994) have defined an outlier in a set of data to be "an observation" or subset of observations" which appears to be inconsistent with the remainder of the set of data". They also describe several models for outlier; see (Moshref and Sultan, 2007). Density functions and joint density functions of order statistics arising from a sample of a single outlier have been given by Shu (1978) and Hartley and David (1978). One may also refer to Vaughan and Venables (1972) for more general expressions of distributions of order statistics using permanent expressions. Arnold and Balakrishnan (1989) have obtained the density function of Xr:n when the sample of size $n$ contains unidentified 
single outlier. They also obtained the joint density function of $X_{\mathrm{r}: \mathrm{n}}$ and $\mathrm{X}_{\mathrm{s}: \mathrm{n}}, 1 \leq \mathrm{r}<\mathrm{s} \leq \mathrm{n}$. Balakrishnan and Balasubramanian (1995) has derived some recurrence relations satisfied by the single and product moments of order statistics from the right truncated exponential distribution. Also he has deduced the recurrence relations for the multiple outlier models (with slippage of observations), see also Balakrishnan (1994). Childs et al. (2001) have derived some recurrence relations for the single and product moments of order statistics from $n$ independent and non-identically distributed Lomax and the right-truncated Lomax random variables.

We assume $X_{1}, X_{2}, \ldots, X_{p}$ are independent with probability density function $f(x)$ while $X_{n}-p+1, \ldots, X_{n}$ are independent were arise from some modified version of $f(x)$ which call $g(x)$ in which the shape parameters have been shifted in value. Finally, some special cases are deduced.

The probability density function of the rth order statistics $X_{\mathrm{r}: \mathrm{n}}$, under the multiple outlier model can be written as, see Childs (1996) Equation 1:

$$
\begin{aligned}
& \mathrm{f}_{\mathrm{r}: \mathrm{n}}[\mathrm{p}](\mathrm{x})=\sum_{\mathrm{s}=\max (0, \mathrm{r}-\mathrm{p}-1)}^{\min (\mathrm{n}-\mathrm{p}-1, \mathrm{r}-1)} \\
& \mathrm{C}_{1} \mathrm{f}(\mathrm{x})\{\mathrm{F}(\mathrm{x})\}^{\mathrm{s}}\{\mathrm{G}(\mathrm{x})\}^{\mathrm{r}-\mathrm{s}-1} \\
& x\{1-F(x)\}^{n-p-s-1}\{1-G(x)\}^{p-r+s+1} \\
& +\sum_{\mathrm{s}=\max (0, \mathrm{r}-\mathrm{p})}^{\min (\mathrm{n}-\mathrm{p}, \mathrm{r}-1)} \\
& \mathrm{C}_{2} \mathrm{~g}(\mathrm{x})\{\mathrm{F}(\mathrm{x})\}^{\mathrm{s}}\{\mathrm{G}(\mathrm{x})\}^{\mathrm{r}-\mathrm{s}-1} \\
& \times\{1-F(x)\}^{n-p-s}\{1-G(x)\}^{p-r+s} \text {, } \\
& 1 \leq \mathrm{r} \leq \mathrm{n}, \mathrm{p}=0,1,2, \cdots \mathrm{n},-\infty<\mathrm{x}<\infty
\end{aligned}
$$

Where Equation 2:

$$
\begin{aligned}
& \mathrm{C}_{1}=\frac{(\mathrm{n}-\mathrm{p}) ! \mathrm{p} !}{\mathrm{s} !(\mathrm{r}-\mathrm{s}-1) !(\mathrm{n}-\mathrm{p}-\mathrm{s}-1) !(\mathrm{p}-\mathrm{r}+\mathrm{s}+1)} \\
& \mathrm{C}_{2}=\frac{(\mathrm{n}-\mathrm{p}) ! \mathrm{p} !}{\mathrm{s} !(\mathrm{r}-\mathrm{s}-1) !(\mathrm{n}-\mathrm{p}-\mathrm{s}) !(\mathrm{p}-\mathrm{r}+\mathrm{s})}
\end{aligned}
$$

Setting $p=1$ in (1) we obtain the corresponding pdf's in case of the single outlier given by Shu (1978).

In this study, we consider the case when the variable $X_{1}, X_{2}, \ldots, X_{n-p}$ are independent observations from Burr XII with four parameters distribution with density Equation 3:

$$
\begin{aligned}
& f(x)=\frac{\rho c}{b}\left(1+\left(\frac{x-a}{b}\right)^{c}\right)^{-\rho-1} \\
& \left(\frac{x-a}{b}\right)^{c-1}, \\
& a \leq x \leq \infty, \rho>0, c>0, b>0
\end{aligned}
$$

and $\mathrm{X}_{\mathrm{n}-\mathrm{p}+1}, \ldots, \mathrm{X}_{\mathrm{n}}$ arise from the same distribution with density Equation 4:

$$
\begin{aligned}
& \mathrm{g}(\mathrm{x})=\frac{\tau \mathrm{c}}{\mathrm{b}}\left(1+\left(\frac{\mathrm{x}-\mathrm{a}}{\mathrm{b}}\right)^{\mathrm{c}}\right)^{-\tau-1} \\
& \left(\frac{\mathrm{x}-\mathrm{a}}{\mathrm{b}}\right)^{\mathrm{c}-1}, \\
& \mathrm{a} \leq \mathrm{x} \leq \infty, \tau>0, \mathrm{c}>0, \mathrm{~b}>0
\end{aligned}
$$

The corresponding cumulative distribution functions $\mathrm{F}(\mathrm{x})$ and $\mathrm{G}(\mathrm{x})$ are given as Equation 5 and 6:

$$
\begin{aligned}
& F(x)=1-\left(1+\left(\frac{x-a}{b}\right)^{c}\right)^{-\rho}, \\
& x \geq a, \rho>0, c>0, b>0 \\
& G(x)=1-\left(1+\left(\frac{x-a}{b}\right)^{c}\right)^{-\tau}, \\
& a £ x, \tau>0, c>0, b>0
\end{aligned}
$$

The relation between $\mathrm{f}(\mathrm{x})$ and $\mathrm{F}(\mathrm{x})$ is given by Equation 7:

$$
\begin{aligned}
& f(x)=\frac{\rho c}{b}\left(\frac{x-a}{b}\right)^{c-1}[1-F(x)] \\
& -\left(\frac{x-a}{b}\right)^{c} f(x)
\end{aligned}
$$

Similarly, the relation between $\mathrm{g}(\mathrm{x})$ and $\mathrm{G}(\mathrm{x})$ is Equation 8:

$$
\begin{aligned}
& g(x)=\frac{\tau c}{b}\left(\frac{x-a}{b}\right)^{c-1}[1-G(x)] \\
& -\left(\frac{x-a}{b}\right)^{c} g(x)
\end{aligned}
$$

In the following section, we use (3) and (4) to derive the single and product moments of order statistics from Burr XII distribution under the multiple outlier models. This situation is known as a multiple outlier model with 
slippage of p observations; Barnett and Lewise (1994). This specific multiple outlier model was introduced by Launer and Bills (1979).

\subsection{Single Moments}

We derive the $\mathrm{k}_{\mathrm{th}}$ moment of the $\mathrm{r}_{\text {th }}$ order statistic under multiple outlier models (with a slippage of $\mathrm{p}$ observations). Let $\mu(\mathrm{k})[\mathrm{p}] ;(1 \leq \mathrm{r} \leq \mathrm{n})$ denote the $\mathrm{k}_{\mathrm{th}}$ single moments of order statistics in the presence of p-outlier observations from BurrXII distribution The following theorem gives an explicit form of $\mu(\mathrm{k})$ [p].

\section{Theorem 1}

For $1 \leq \mathrm{r} \leq \mathrm{n}, \mathrm{p}=0,1, \ldots, \mathrm{n}$ and $\mathrm{k}=0,1, \ldots$ the single moments $\mu_{\mathrm{r}: \mathrm{n}}^{(\mathrm{k})}[\mathrm{p}]$ is given by Equation 9:

$$
\begin{aligned}
& \therefore \mu_{\mathrm{r}: \mathrm{n}}^{(\mathrm{k})}[\mathrm{p}]=\rho \mathrm{b}^{\mathrm{k}} \sum_{\mathrm{s}=\max (0, \mathrm{r}-\mathrm{p}-1)}^{\min (\mathrm{n}-\mathrm{p}-1, \mathrm{r}-1)} \\
& \mathrm{C}_{1} \sum_{1=0}^{\mathrm{s}}\left(\begin{array}{l}
\mathrm{s} \\
1
\end{array}\right) \sum_{\mathrm{m}=0}^{\mathrm{r}-\mathrm{s}-1}\left(\begin{array}{c}
\mathrm{r}-\mathrm{s}-1 \\
\mathrm{~m}
\end{array}\right)(-1)^{1+\mathrm{m}} \\
& \times \sum_{i=0}^{k}\left(\frac{a}{b}\right)^{i}\left(\begin{array}{c}
k \\
i
\end{array}\right) \beta\left(\phi-\frac{k}{c}+\frac{i}{c}-1, \frac{k}{c}-\frac{i}{c}+1\right) \\
& +\tau b^{k} \sum_{s=\max (0, r-p)}^{\min (n-p, r-1)} \\
& \left.\mathrm{C}_{2} \sum_{1=0}^{\mathrm{s}}\left(\begin{array}{l}
\mathrm{s} \\
1
\end{array}\right)^{\mathrm{r}-\mathrm{r}=0} \sum_{\mathrm{m}-1}^{\mathrm{r}-\mathrm{s}-1} \begin{array}{c}
\mathrm{m}
\end{array}\right)(-1)^{1+\mathrm{m}} \\
& \times \sum_{i=0}^{k}\left(\frac{a}{b}\right)^{i}\left(\begin{array}{c}
k \\
i
\end{array}\right) \beta\left(\phi-\frac{k}{c}+\frac{i}{c}-1, \frac{k}{c}-\frac{i}{c}+1\right)
\end{aligned}
$$

where, $0^{0}=1, b \neq 0$ Equation 10 :

$$
\begin{aligned}
& \phi=\rho(n-p-s+1)+\tau(p-r+s+1+m)+1, \\
& C_{1}=\frac{(n-p) ! p !}{s !(r-s-1) !(n-p-s-1) !(p-r+s+1)} \\
& C_{2}=\frac{(n-p) ! p !}{s !(r-s-1) !(n-p-s) !(p-r+s)}
\end{aligned}
$$

$$
\begin{aligned}
& \{1-\mathrm{F}(\mathrm{x})\}^{\mathrm{n}-\mathrm{p}-\mathrm{s}-1}\{1-\mathrm{G}(\mathrm{x})\}^{\mathrm{p}-\mathrm{r}+\mathrm{s}+1} \mathrm{dx} \\
& \quad+\sum_{\mathrm{s}=\max (0, \mathrm{r}-\mathrm{p})}^{\min (\mathrm{n}-\mathrm{p}, \mathrm{r}-1)} \mathrm{C}_{2} \int_{0}^{\infty} \mathrm{x}^{\mathrm{k}} \mathrm{g}(\mathrm{x})\{\mathrm{F}(\mathrm{x})\}^{\mathrm{s}}\{\mathrm{G}(\mathrm{x})\}^{\mathrm{r}-\mathrm{s}-1} \\
& \{1-\mathrm{F}(\mathrm{x})\}^{\mathrm{n}-\mathrm{p}-\mathrm{s}}\{1-\mathrm{G}(\mathrm{x})\}^{\mathrm{p}-\mathrm{r}+\mathrm{s}} \mathrm{dx}
\end{aligned}
$$

Using the relations Equation 12:

$$
\begin{gathered}
{[1-F(x)]^{-1}=\frac{\frac{\rho c}{b}\left(\frac{x-a}{b}\right)^{c-1}}{f(x)\left(1+\left(\frac{x-a}{b}\right)^{c}\right)}} \\
g(x)=\frac{\frac{\tau c}{b}[1-G(x)]\left(\frac{x-a}{b}\right)^{c-1}}{\left(1+\left(\frac{x-a}{b}\right)^{c}\right)}
\end{gathered}
$$

We get Equation 13 and 14:

$$
\begin{aligned}
& \mu_{\mathrm{r}: \mathrm{n}}^{(\mathrm{k})}[\mathrm{p}]=\sum_{\mathrm{s}=\max (0, \mathrm{r}-\mathrm{p}-1)}^{\min (\mathrm{n}-\mathrm{p}-1, \mathrm{r}-1)} \mathrm{C}_{1} \int_{\mathrm{a}}^{\infty} \\
& x^{k} f(x)\{F(x)\}^{s}\{G(x)\}^{r-s-1} \\
& \{1-\mathrm{F}(\mathrm{x})\}^{\mathrm{n}-\mathrm{p}-\mathrm{s}} \\
& \times \frac{\frac{\rho c}{b}\left(\frac{x-a}{b}\right)^{c-1}}{f(x)\left(1+\left(\frac{x-a}{b}\right)^{c}\right)} \\
& \{1-\mathrm{G}(\mathrm{x})\}^{\mathrm{p}-\mathrm{r}+\mathrm{s}+1} \mathrm{dx} \\
& +\sum_{\mathrm{s}=\max (0, r-p)}^{\min (\mathrm{n}-\mathrm{p}, \mathrm{r}-\mathrm{p})} \mathrm{C}_{2} \int_{\mathrm{a}}^{\infty} \\
& x^{k} \frac{\frac{\tau c}{b}[1-G(x)]\left(\frac{x-a}{b}\right)^{c-1}}{\left(1+\left(\frac{x-a}{b}\right)^{c}\right)} \\
& \{\mathrm{F}(\mathrm{x})\}^{\mathrm{s}}\{\mathrm{G}(\mathrm{x})\}^{\mathrm{r}-\mathrm{s}-1} \\
& \times\{1-\mathrm{F}(\mathrm{x})\}^{\mathrm{n}-\mathrm{p}-\mathrm{s}}\{1-\mathrm{G}(\mathrm{x})\}^{\mathrm{p}-\mathrm{r}+\mathrm{s}} \mathrm{dx} \\
& \mu_{\mathrm{r}: \mathrm{n}}^{(\mathrm{k})}[\mathrm{p}]=\frac{\rho \mathrm{c}}{\mathrm{b}^{\mathrm{c}}} \sum_{\mathrm{s}=\max (0, \mathrm{r}-\mathrm{p}-1)}^{\min (\mathrm{n}-\mathrm{p}-1, \mathrm{r}-1)} \\
& \mathrm{C}_{1} \int_{\mathrm{a}}^{\infty} \mathrm{x}^{\mathrm{k}}(\mathrm{x}-\mathrm{a})^{\mathrm{c}-1}\left(1+\left(\frac{\mathrm{x}-\mathrm{a}}{\mathrm{b}}\right)^{\mathrm{c}}\right)^{-1} \\
& \{F(x)\}^{\mathrm{s}}\{\mathrm{G}(\mathrm{x})\}^{\mathrm{r}-\mathrm{s}-1} \times \\
& \{1-\mathrm{F}(\mathrm{x})\}^{\mathrm{n}-\mathrm{p}-\mathrm{s}}\{1-\mathrm{G}(\mathrm{x})\}^{\mathrm{p}-\mathrm{r}+\mathrm{s}+1} \mathrm{dx} \\
& +\frac{\tau c}{b^{C}} \sum_{s=\max (0, r-p)}^{\min (n-p, r-1)} \\
& \mathrm{C}_{2} \int_{\mathrm{a}}^{\infty} \mathrm{x}^{\mathrm{k}}(\mathrm{x}-\mathrm{a})^{\mathrm{c}-1}\left(1+\left(\frac{\mathrm{x}-\mathrm{a}}{\mathrm{b}}\right)^{\mathrm{c}}\right)^{-1} \\
& \{\mathrm{~F}(\mathrm{x})\}^{\mathrm{s}}\{\mathrm{G}(\mathrm{x})\}^{\mathrm{r}-\mathrm{s}-1} \times\{1-\mathrm{F}(\mathrm{x})\}^{\mathrm{n}-\mathrm{p}-\mathrm{s}} \\
& \{1-\mathrm{G}(\mathrm{x})\}^{\mathrm{p}-\mathrm{r}+\mathrm{s}+1} \mathrm{dx}
\end{aligned}
$$

Now by writing: 
$\mathrm{F}(\mathrm{x})=1-(1-\mathrm{F}(\mathrm{x}))$ and $\mathrm{G}(\mathrm{x})=1-(1-\mathrm{G}(\mathrm{x}))$ in (14) and expand we get Equation 15:

$$
\begin{aligned}
& \mu_{r: n}^{(k)}[p]=\frac{\rho c}{b^{c}} \sum_{s=\max (0, r-p-1)}^{\min (n-p-1, r-1)} \\
& C_{1} \int_{a}^{\infty} x^{k}(x-a)^{c-1}\left(1+\left(\frac{x-a}{b}\right)^{c}\right)^{-1} \\
& \sum_{\mathrm{l}=0}^{\mathrm{s}}\left(\begin{array}{l}
\mathrm{s} \\
1
\end{array}\right) \sum_{\mathrm{m}=0}^{\mathrm{r}-\mathrm{s}-1}\left(\begin{array}{c}
\mathrm{r}-\mathrm{s}-1 \\
\mathrm{~m}
\end{array}\right)(-1)^{1+\mathrm{m}} \\
& x\{1-F(x)\}^{n-p-s+1} \\
& \{1-\mathrm{G}(\mathrm{x})\}^{\mathrm{p}-\mathrm{r}+\mathrm{s}+1+\mathrm{m}} \mathrm{dx} \\
& +\frac{\tau \mathrm{C}}{\mathrm{b}^{\mathrm{C}}} \sum_{\mathrm{s}=\max (0, \mathrm{~m}-\mathrm{p})}^{\min (\mathrm{n}-\mathrm{p}, \mathrm{r}-\mathrm{l})} \mathrm{C}_{2} \int_{\mathrm{a}}^{\infty} \\
& x^{k}(x-a)^{c-1}\left(1+\left(\frac{x-a}{b}\right)^{c}\right)^{-1} \sum_{1=0}^{s} \\
& \left(\begin{array}{l}
\mathrm{s} \\
1
\end{array}\right)_{\mathrm{m}=0}^{\mathrm{r}-\mathrm{s}-1}\left(\begin{array}{c}
\mathrm{r}-\mathrm{s}-1 \\
\mathrm{~m}
\end{array}\right)(-1)^{1+\mathrm{m}} \\
& x\{1-F(x)\}^{n-p-s+1} \\
& \{1-\mathrm{G}(\mathrm{x})\}^{\mathrm{p}-\mathrm{r}+\mathrm{s}+1+\mathrm{m}} \mathrm{dx}
\end{aligned}
$$

We know that Equation 16-18:

$$
\begin{aligned}
& 1-F(x)=\left(1+\left(\frac{x-a}{b}\right)^{c}\right)^{-\rho} 1-G(x)=\left(1+\left(\frac{x-a}{b}\right)^{c}\right)^{-\tau} \\
& \mu_{\mathrm{r}: \mathrm{n}}^{(\mathrm{k})}[\mathrm{p}]=\frac{\rho \mathrm{c}}{\mathrm{b}^{\mathrm{c}}}{ }_{\mathrm{s}=\max (0, \mathrm{r}-\mathrm{p}-1)}^{\min (\mathrm{n}-\mathrm{p}-1, \mathrm{r}-1)} \\
& \mathrm{C}_{1} \int_{\mathrm{a}}^{\infty} \mathrm{x}^{\mathrm{k}}(\mathrm{x}-\mathrm{a})^{\mathrm{c}-1} \sum_{\mathrm{l}=0}^{\mathrm{s}} \\
& \left(\begin{array}{l}
\mathrm{s} \\
1
\end{array}\right)_{\mathrm{m}=0}^{\mathrm{r}-\mathrm{s}-1}\left(\begin{array}{c}
\mathrm{r}-\mathrm{s}-1 \\
\mathrm{~m}
\end{array}\right)(-1)^{1+\mathrm{m}} \\
& -[\rho(n-p-s+1) \mid+\tau
\end{aligned}
$$

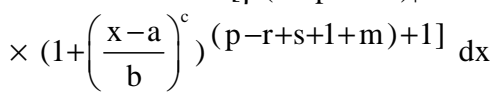

$$
\begin{aligned}
& +\frac{\tau c}{b^{c}} \sum_{s=\max (0, r-p)}^{\min (n-p, r-1)} C_{2} \int_{a}^{\infty} \\
& x^{k}(x-a)^{c-1} \sum_{i=0}^{s} \\
& \left(\begin{array}{l}
\mathrm{s} \\
1
\end{array}\right)_{\mathrm{m}=0}^{\mathrm{r}-\mathrm{s}-1}\left(\begin{array}{c}
\mathrm{r}-\mathrm{s}-1 \\
\mathrm{~m}
\end{array}\right)(-1)^{1+\mathrm{m}} \\
& -[\rho(n-p-s+1) \mid+\tau \\
& \times\left(1+\left(\frac{x-a}{b}\right)^{c}\right)^{(p-r+s+1+m)+1]} d x
\end{aligned}
$$

$$
\begin{aligned}
& \frac{1}{\mathrm{y}}=1+\left(\frac{\mathrm{x}-\mathrm{a}}{\mathrm{b}}\right)^{\mathrm{c}} \Rightarrow\left(\frac{\mathrm{x}-\mathrm{a}}{\mathrm{b}}\right)^{\mathrm{c}}=\frac{1-\mathrm{y}}{\mathrm{y}} \Rightarrow \\
& \left(\frac{x-a}{b}\right)=\left(\frac{1-y}{y}\right)^{\frac{1}{c}} \Rightarrow x-a=b\left(\frac{1-y}{y}\right)^{\frac{1}{c}} \\
& \therefore \mathrm{x}=\mathrm{b}\left(\frac{1-\mathrm{y}}{\mathrm{y}}\right)^{\frac{1}{\mathrm{c}}}+\mathrm{a} \\
& \mathrm{dx}=\frac{\mathrm{b}}{\mathrm{c}}\left(\frac{1-\mathrm{y}}{\mathrm{y}}\right)^{\frac{1}{\mathrm{c}^{-1}}}\left(-\frac{1}{\mathrm{y}^{2}}\right) \mathrm{dy} \\
& \text { at } x=a \Rightarrow y=1 \text {, at } x=\infty \Rightarrow y=0 \\
& \mu_{\mathrm{r}: \mathrm{n}}^{(\mathrm{k})}[\mathrm{p}]=\rho \mathrm{b}^{\mathrm{k}} \sum_{\mathrm{s}=\max (0, \mathrm{r}-\mathrm{p}-1)}^{\min (\mathrm{n}-\mathrm{p}-1, \mathrm{r}-\mathrm{l})} \\
& \mathrm{C}_{1} \sum_{\mathrm{l}=0}^{\mathrm{s}}\left(\begin{array}{l}
\mathrm{s} \\
1
\end{array}\right) \sum_{\mathrm{m}=0}^{\mathrm{r}-\mathrm{s}-1}\left(\begin{array}{c}
\mathrm{r}-\mathrm{s}-1 \\
\mathrm{~m}
\end{array}\right)(-1)^{1+\mathrm{m}} \\
& \times \int_{0}^{1}\left[\mathrm{~b}\left(\frac{1-\mathrm{y}}{\mathrm{y}}\right)^{\frac{1}{\mathrm{c}}}+\mathrm{a}\right]^{\mathrm{k}} \\
& {[\rho(\mathrm{n}-\mathrm{p}-\mathrm{s}+1)} \\
& x \mathrm{y}^{+\tau(\mathrm{p}-\mathrm{r}+\mathrm{s}+1+\mathrm{m})+1]-2} d \mathrm{y} \\
& +\tau \mathrm{b}^{\mathrm{k}} \sum_{\mathrm{s}=\max (0, \mathrm{r}-\mathrm{p})}^{\min (\mathrm{n}-\mathrm{p}, \mathrm{r}-1)} \\
& \mathrm{C}_{2} \sum_{\mathrm{l}=2}^{\mathrm{s}}\left(\begin{array}{l}
\mathrm{s} \\
1
\end{array}\right) \sum_{\mathrm{m}=0}^{\mathrm{r}-\mathrm{s}-1}\left(\begin{array}{c}
\mathrm{r}-\mathrm{s}-1 \\
\mathrm{~m}
\end{array}\right)(-1)^{1+\mathrm{m}} \\
& \times \int_{0}^{1}\left[\mathrm{~b}\left(\frac{1-\mathrm{y}}{\mathrm{y}}\right)^{\frac{1}{\mathrm{c}}}+\mathrm{a}\right]^{\mathrm{k}} \\
& {[\rho(n-p-s+1) \mid+\tau} \\
& \times y^{(p-r+s+1+m)+11-2} d y
\end{aligned}
$$

Let:

$$
\begin{aligned}
& {\left[b\left(\frac{1-y}{y}\right)^{\frac{1}{c}}+a\right]^{k}} \\
& =\sum_{i=0}^{k}\left(\begin{array}{l}
k \\
i
\end{array}\right) a^{i}\left(\frac{1-y}{y}\right)^{\frac{k-i}{c}} b^{k=i}
\end{aligned}
$$

$$
\text { Le: }
$$$$
\therefore \mu_{\mathrm{r}: \mathrm{n}}^{(\mathrm{k})}[\mathrm{p}]=\rho \mathrm{b}^{\mathrm{k}} \sum_{\mathrm{s}=\max (0, \mathrm{r}-\mathrm{p}-1)}^{\min (\mathrm{n}-\mathrm{p}-1, \mathrm{r}-1)}
$$ 


$$
\begin{aligned}
& \mathrm{C}_{1} \sum_{1-0}^{\mathrm{s}}\left(\begin{array}{l}
\mathrm{s} \\
1
\end{array}\right) \sum_{\mathrm{m}=0}^{\mathrm{r}-\mathrm{s}-1}\left(\begin{array}{c}
\mathrm{r}-\mathrm{s}-1 \\
\mathrm{~m}
\end{array}\right)(-1)^{1+\mathrm{m}} \\
& \times \sum_{\mathrm{i}=1}^{\mathrm{k}}\left(\frac{\mathrm{a}}{\mathrm{b}}\right)^{\mathrm{i}}\left(\begin{array}{c}
\mathrm{k} \\
\mathrm{i}
\end{array}\right) \int_{0}^{1}(1-\mathrm{y})^{\frac{\mathrm{k}-\mathrm{i}}{\mathrm{c}}} \\
& {[\rho(n-p-s+1) \mid+} \\
& \tau(\mathrm{p}-\mathrm{r}+\mathrm{s}+1+\mathrm{m})+1]-\frac{\mathrm{k}-\mathrm{i}}{\mathrm{c}}-2 \\
& +\tau \mathrm{b}^{\mathrm{k}} \sum_{\mathrm{s}=\max (0, \mathrm{r}-\mathrm{p})}^{\min (\mathrm{n}-\mathrm{p}, \mathrm{r}-1)} \\
& \mathrm{C}_{2} \sum_{1=0}^{\mathrm{s}}\left(\begin{array}{l}
\mathrm{s} \\
1
\end{array}\right) \sum_{\mathrm{m}=0}^{\mathrm{r}-\mathrm{s}-1}\left(\begin{array}{c}
\mathrm{r}-\mathrm{s}-1 \\
\mathrm{~m}
\end{array}\right)(-1)^{1+\mathrm{m}} \\
& \times \sum_{i=0}^{k}\left(\frac{a}{b}\right)^{i}\left(\begin{array}{l}
k \\
i
\end{array}\right) \int_{0}^{1}(1-y)^{\frac{k-i}{c}} \\
& {[\rho(n-p-s+1) \mid+} \\
& y^{\tau(p-r+s+1+m)+1]-\frac{k-i}{c}-2} d y
\end{aligned}
$$

Let $\Phi=\rho(n-p-s+1)+\tau(p-r+s+1+m)+1$ Equation 19 :

$$
\begin{aligned}
& \int_{0}^{1}(1-\mathrm{y})^{\frac{\mathrm{k}-\mathrm{i}}{\mathrm{c}}} \mathrm{y}{ }^{\Phi-\frac{\mathrm{k}-\mathrm{i}}{\mathrm{c}}-2} \mathrm{dy} \\
& =\beta\left(\Phi-\frac{\mathrm{k}-\mathrm{i}}{\mathrm{c}}-2, \frac{\mathrm{k}-\mathrm{i}}{\mathrm{c}}+1\right) \\
& \therefore \mu_{\mathrm{r}: \mathrm{n}}^{(\mathrm{k})}[\mathrm{p}]=\rho \mathrm{b}^{\mathrm{k}} \sum_{\mathrm{m}=\max (0, \mathrm{r}-\mathrm{p}-1)}^{(\mathrm{n}-\mathrm{p}-1, \mathrm{r}-1)} \\
& \mathrm{C}_{1} \sum_{\mathrm{l}=0}^{\mathrm{s}}\left(\frac{\mathrm{s}}{1}\right)^{\mathrm{r}-\mathrm{s}-1} \sum_{\mathrm{m}=0}^{\mathrm{r}-1}\left(\begin{array}{c}
\mathrm{r}-\mathrm{s}-1 \\
\mathrm{~m}
\end{array}\right)(-1)^{1+\mathrm{m}} \\
& \times \sum_{\mathrm{i}=0}^{\mathrm{k}}\left(\frac{\mathrm{a}}{\mathrm{b}}\right)^{\mathrm{i}}\left(\begin{array}{l}
\mathrm{k} \\
\mathrm{i}
\end{array}\right) \beta\left(\phi-\frac{\mathrm{k}}{\mathrm{c}}+\frac{\mathrm{i}}{\mathrm{c}}-1, \frac{\mathrm{k}}{\mathrm{c}}-\frac{\mathrm{i}}{\mathrm{c}}+1\right) \\
& +\tau \mathrm{b}^{\mathrm{k}} \sum_{\mathrm{min}(\mathrm{n}-\mathrm{p}, \mathrm{r}-1)}^{\mathrm{s}=\max (0, \mathrm{r}-\mathrm{p})} \mathrm{C}_{2} \sum_{\mathrm{l}=0}^{\mathrm{s}}\left(\begin{array}{l}
\mathrm{s} \\
1
\end{array}\right) \sum_{\mathrm{m}=0}^{\mathrm{r}-\mathrm{s}-1} \\
& \left(\begin{array}{c}
\mathrm{r}-\mathrm{s}-1 \\
\mathrm{~m}
\end{array}\right)(-1)^{1+\mathrm{m}} \\
& \times \sum_{\mathrm{i}-0}^{\mathrm{k}}\left(\frac{\mathrm{a}}{\mathrm{b}}\right)^{\mathrm{i}}\left(\begin{array}{l}
\mathrm{k} \\
\mathrm{i}
\end{array}\right) \beta\left(\phi-\frac{\mathrm{k}}{\mathrm{c}}+\frac{\mathrm{i}}{\mathrm{c}}-1, \frac{\mathrm{k}}{\mathrm{c}}-\frac{\mathrm{i}}{\mathrm{c}}+1\right)
\end{aligned}
$$

\section{Remark}

If we put $\mathrm{a}=0, \mathrm{~b}=1$ in (9) and consider $0^{0}=1$ we get moments of order statistics from Burr XII distribution with two parameters in the presence of outlier observations as Equation 20:

$$
\begin{aligned}
& \therefore \mu_{\mathrm{r}: \mathrm{n}}^{(\mathrm{k})}[\mathrm{p}]=\rho \sum_{\mathrm{s}=\max (0, \mathrm{r}-\mathrm{p}-1)}^{\min (\mathrm{n}-\mathrm{p}-1, \mathrm{r}-1)} \\
& \mathrm{C}_{1} \sum_{\mathrm{l}=0}^{\mathrm{s}}\left(\begin{array}{l}
\mathrm{s} \\
1
\end{array}\right) \sum_{\mathrm{m}=0}^{\mathrm{r}-\mathrm{s}-1}\left(\begin{array}{c}
\mathrm{r}-\mathrm{s}-1 \\
\mathrm{~m}
\end{array}\right) \\
& (-1)^{1+\mathrm{m}} \beta\left(\phi-\frac{\mathrm{k}}{\mathrm{c}}-1, \frac{\mathrm{k}}{\mathrm{c}}+1\right) \\
& +\tau \sum_{\mathrm{m}=\max (0, \mathrm{r}-\mathrm{p})}^{\min (\mathrm{n}-\mathrm{p}, \mathrm{r}-1)} \mathrm{C}_{2} \sum_{1=0}^{\mathrm{s}}\left(\begin{array}{l}
\mathrm{s} \\
1
\end{array}\right) \sum_{\mathrm{m}=0}^{\mathrm{r}-\mathrm{s}-1} \\
& \left(\begin{array}{c}
\mathrm{r}-\mathrm{s}-1 \\
\mathrm{~m}
\end{array}\right)(-1)^{1+\mathrm{m}} \beta\left(\phi-\frac{\mathrm{k}}{\mathrm{c}}-1, \frac{\mathrm{k}}{\mathrm{c}}+1\right)
\end{aligned}
$$

\section{Special Cases}

We deduce some special cases from the single moments given in (9) and (20) as follows:

- Setting $p=0$, we get the single moments of order statistics when $\mathrm{x}_{1}, \mathrm{x}_{2}, \ldots, \mathrm{x}_{\mathrm{n}}$ have Burr distribution as Equation 21 and 22:

$$
\begin{aligned}
& \mu_{r: n}^{(k)}[0]=\frac{\rho b^{k} n !}{(r-1) !(n-r) !} \sum_{1=0}^{r-1}\left(\begin{array}{c}
r-1 \\
1
\end{array}\right) \\
& (-1)^{1} \sum_{i=0}^{k}\left(\frac{a}{b}\right)^{i}\left(\begin{array}{c}
k \\
i
\end{array}\right) \\
& \beta\left(\phi-\frac{k}{c}+\frac{i}{c}-1, \frac{k}{c}-\frac{i}{c}+1\right) \\
& \Phi=\rho(n-r+1+1)+1 \\
& \mu_{r: n}^{(k)}[0]=\frac{\rho n !}{(r-1) !(n-r) !} \sum_{1=0}^{r-1}\left(\begin{array}{c}
r-1 \\
1
\end{array}\right) \\
& (-1)^{1} \beta\left(\phi-\frac{k}{c}+\frac{i}{c}-1, \frac{k}{c}-\frac{i}{c}+1\right) \\
& \Phi=\rho(n-r+1+1)+1
\end{aligned}
$$

- If we put $p=n$, we have the same relations above but with parameter $\tau$

- If we put $p=1$, we have the relations for single outlier

\section{Example (1)}

Let $\mathrm{X}_{1}, \mathrm{X}_{2} \sim$ Barr XII with $\mathrm{c}=1.39533, \rho=3$, $\mathrm{a}=$ $0.13445, b=15.67968$ and $X_{3} \sim \operatorname{Gamma}(\beta=1, \alpha=2)$. Find $\mu_{1: 3}$.

\section{Solution}

A single outlier in this sample that $\mathrm{X}_{3} \sim \operatorname{Gamma}(\beta=$ $1, \alpha=2)$. 
Jamjoom, A.A. and Z.A. Al-Saiary /Journal of Mathematics and Statistics 9 (1): 51-61, 2013

Table 1. Results of Tadikamalla (1977) for the parameters $\rho, a, b, c, \mu, \sigma$ when $\alpha$ is integer and $\beta=1$

\begin{tabular}{lrlrlrl}
\hline$\alpha$ & $\mathrm{k}$ & $\mathrm{c}$ & $\mathrm{a}$ & $\mathrm{b}$ & $\mu$ & $\sigma$ \\
\hline 2.0 & 17.97966 & 1.39533 & 0.13445 & 15.67968 & 2.001763475 & 1.415469000 \\
$* 3.0$ & 11.87409 & 1.66677 & 0.34929 & 12.56702 & 3.001531924 & 1.733370325 \\
4.0 & 9.81594 & 1.87242 & 0.62394 & 12.34255 & 4.002643944 & 2.001365465 \\
$* 5.0$ & 8.78044 & 2.03679 & 0.94586 & 12.74163 & 5.003001406 & 2.237449340 \\
6.0 & 8.15747 & 2.17284 & 1.30639 & 13.34974 & 6.003306453 & 2.450905466 \\
8.0 & 7.44596 & 2.38816 & 2.12026 & 14.75852 & 8.003844356 & 2.829869466 \\
10.0 & -7.05312 & 2.55363 & 3.03161 & 16.21132 & 10.004322450 & 3.163752577 \\
15 & 6.57114 & 2.84543 & 5.61319 & 19.67139 & 15.011104120 & 3.874534021 \\
25 & -6.22985 & 3.18708 & 11.58794 & 25.68629 & 24.999992660 & 4.999998172 \\
50 & 6.02306 & 3.58956 & 28.87270 & 37.49438 & 49.999986320 & 7.071055695 \\
100 & 5.96191 & 3.91545 & 67.62502 & 54.86029 & 100.000013700 & 9.999999494 \\
$\infty$ Normal & 6.15784 & 4.87370 & -3.97998 & 6.17322 & $-1.21 \mathrm{E}-07000$ & 0.999999908 \\
\hline
\end{tabular}

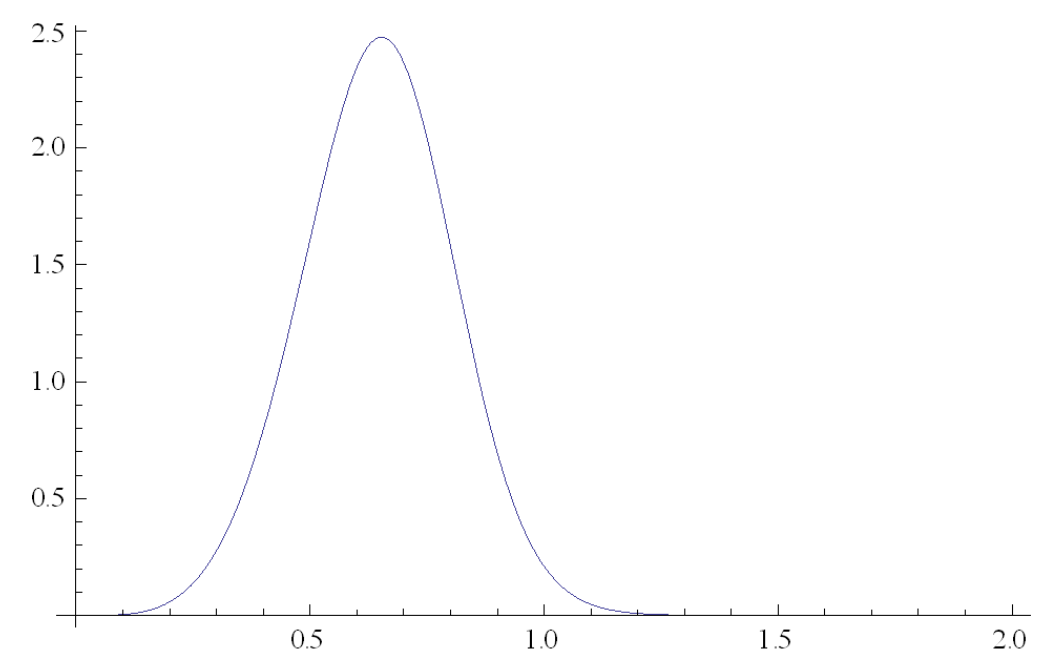

Fig 1. Burr distribution with $(\mathrm{c}=4.8773717, \tau=6.157568)$

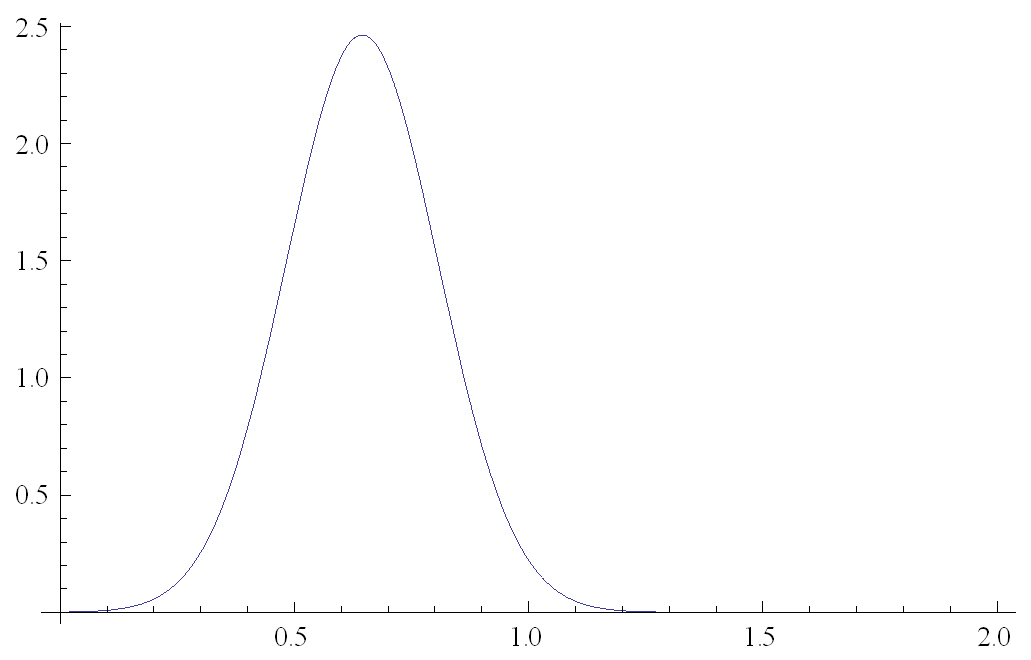

Fig 2. Normal distribution with $(\mu=0.644717, \sigma=0.16199)$ 
But we know that Tadikamalla (1977) approximate Gamma distribution $\beta=1, \alpha=2$ with Burr XII with four parameters as $c=1.39533, \rho=17.97966, \mathrm{a}=0.13445, \mathrm{~b}$ $=15.67968$ Table 1 and Fig. 1 and 2 . So when $\mathrm{X}_{3} \sim$ Gamma $(\beta=1, \alpha=2) \Rightarrow X_{3} \sim$ Burr IIX with parameter $(\mathrm{c}=1.39533, \rho=17.97966, \mathrm{a}=0.13445, \mathrm{~b}=$ 15.67968).

Now using Equation 9:

$$
\begin{gathered}
\mu_{1: 3}^{(\mathrm{k})}[1]=3(15.67968)^{\mathrm{k}} \sum_{\mathrm{s}=\max (0,-1)}^{\min (1,0)} \\
\mathrm{C}_{1} \sum_{1=0}^{\mathrm{s}}\left(\begin{array}{l}
\mathrm{s} \\
1
\end{array}\right) \sum_{\mathrm{m}=0}^{\mathrm{r}-\mathrm{s}-1}\left(\begin{array}{c}
\mathrm{r}-\mathrm{s}-1 \\
\mathrm{~m}
\end{array}\right)(-1)^{1+\mathrm{m}} \\
\times \sum_{\mathrm{i}=0}^{1}\left(\frac{0.13445}{15.67968}\right)^{\mathrm{i}}\left(\begin{array}{l}
\mathrm{k} \\
\mathrm{i}
\end{array}\right)
\end{gathered}
$$$$
\beta\left(\phi-\frac{\mathrm{k}}{1.39533}+\frac{\mathrm{i}}{1.39533}-1, \frac{\mathrm{k}}{1.39533}-\frac{\mathrm{i}}{1.39533}+1\right)
$$$$
+17.97966(15.67968)^{\mathrm{k}} \sum_{\mathrm{s}=\max (0,0)}^{\min (2,0)}
$$$$
\mathrm{C}_{2} \sum_{\mathrm{l}=0}^{\mathrm{s}}\left(\begin{array}{l}
\mathrm{s} \\
1
\end{array}\right)_{\mathrm{m}=0}^{\mathrm{r}-\mathrm{s}-1}\left(\begin{array}{c}
\mathrm{r}-\mathrm{s}-1 \\
\mathrm{~m}
\end{array}\right)(-1)^{1+\mathrm{m}} \times \sum_{\mathrm{i}=0}^{1}\left(\frac{0.13445}{15.67968}\right)^{\mathrm{i}}\left(\begin{array}{l}
\mathrm{k} \\
\mathrm{i}
\end{array}\right)
$$$$
\beta\left(\phi-\frac{\mathrm{k}}{1.39533}+\frac{\mathrm{i}}{1.39533}-1, \frac{\mathrm{k}}{1.39533}-\frac{\mathrm{i}}{1.39533}+1\right)
$$$$
\Phi=3(3-1-\mathrm{s}+1)+(17.97966)(1-1+\mathrm{s}+1+\mathrm{m})
$$$$
+1=3(2-\mathrm{s})+(17.97966)(\mathrm{s}+\mathrm{m}+1)+1
$$$$
\mu_{1: 3}^{(\mathrm{k})}[1]=3(15.67968)^{\mathrm{k}} \sum_{\mathrm{s}=\max (0,-1)}^{\min (1,0)}
$$$$
\mathrm{C}_{1} \sum_{1=0}^{\mathrm{s}}\left(\begin{array}{c}
\mathrm{s} \\
1
\end{array}\right) \sum_{\mathrm{m}=0}^{\mathrm{r}-\mathrm{s}-1}\left(\begin{array}{c}
\mathrm{r}-\mathrm{s}-1 \\
\mathrm{~m}
\end{array}\right)(-1)^{1+\mathrm{m}}
$$$$
\times \sum_{i=0}^{k}\left(\frac{0.13445}{15.67968}\right)^{i}\left(\begin{array}{l}
k \\
i
\end{array}\right)
$$$$
\beta\left(\phi-\frac{\mathrm{k}}{1.39533}+\frac{\mathrm{i}}{1.39533}-1, \frac{\mathrm{k}}{1.39533}-\frac{\mathrm{i}}{1.39533}+1\right)
$$$$
+17.97966(15.67968)^{\mathrm{k}} \sum_{\mathrm{s}=\max (0,0)}^{\min (2,0)}
$$$$
\mathrm{C}_{2} \sum_{1=0}^{\mathrm{s}}\left(\begin{array}{l}
\mathrm{s} \\
1
\end{array}\right) \sum_{\mathrm{m}=0}^{\mathrm{r}-\mathrm{s}-1}\left(\begin{array}{c}
\mathrm{r}-\mathrm{s}-1 \\
\mathrm{~m}
\end{array}\right)(-1)^{1+\mathrm{m}}
$$$$
\times \sum_{i=0}^{k}\left(\frac{0.13445}{15.67968}\right)^{i}\left(\begin{array}{l}
k \\
i
\end{array}\right)
$$$$
\beta\left(\phi-\frac{\mathrm{k}}{1.39533}+\frac{\mathrm{i}}{1.39533}-1, \frac{\mathrm{k}}{1.39533}-\frac{\mathrm{i}}{1.39533}+1\right)
$$$$
\mu_{1: 3}^{(\mathrm{k})}[1]=3(15.67968)^{\mathrm{k}} \sum_{\mathrm{s}=0}^{0} \mathrm{C}_{1} \sum_{\mathrm{l}=0}^{\mathrm{s}}\left(\begin{array}{l}
\mathrm{s} \\
1
\end{array}\right) \sum_{\mathrm{m}=0}^{1-\mathrm{s}-1}\left(\begin{array}{c}
1-\mathrm{s}-1 \\
\mathrm{~m}
\end{array}\right)(-1)^{1+\mathrm{m}}
$$

$$
\begin{aligned}
& \times \sum_{\mathrm{i}=0}^{\mathrm{k}}\left(\frac{0.13445}{15.67968}\right)^{\mathrm{i}}\left(\begin{array}{l}
\mathrm{k} \\
\mathrm{i}
\end{array}\right) \beta\left(\phi-\frac{\mathrm{k}}{1.39533}+\frac{\mathrm{i}}{1.39533}\right. \\
& \left.-1, \frac{\mathrm{k}}{1.39533}-\frac{\mathrm{i}}{1.39533}+1\right)+17.97966(15.67968)^{\mathrm{k}} \\
& \sum_{\mathrm{s}=0}^{0} \mathrm{C}_{2} \sum_{\mathrm{l}=0}^{\mathrm{s}}\left(\begin{array}{l}
\mathrm{s} \\
1
\end{array}\right) \sum_{\mathrm{m}=0}^{1-\mathrm{s}-1}\left(\begin{array}{c}
1-\mathrm{s}-1 \\
\mathrm{~m}
\end{array}\right)(-1)^{1+\mathrm{m}} \times \sum_{\mathrm{i}=0}^{\mathrm{k}}\left(\frac{0.13445}{15.67968}\right)^{\mathrm{i}}\left(\begin{array}{l}
\mathrm{k} \\
\mathrm{i}
\end{array}\right) \\
& \beta\left(\phi-\frac{\mathrm{k}}{1.39533}+\frac{\mathrm{i}}{1.39533}-1, \frac{\mathrm{k}}{1.39533}-\frac{\mathrm{i}}{1.39533}+1\right) \\
& \mu_{1: 3}^{(\mathrm{k})}[1]=3(15.67968)^{\mathrm{k}} \mathrm{C}_{1} \sum_{\mathrm{i}=0}^{\mathrm{k}}\left(\frac{0.13445}{15.67968}\right)^{\mathrm{i}}\left(\begin{array}{l}
\mathrm{k} \\
\mathrm{i}
\end{array}\right) \\
& \times \beta\left(6+(18.97966)-\frac{\mathrm{k}}{1.39533}+\frac{\mathrm{i}}{1.39533}\right. \\
& \left.-1, \frac{\mathrm{k}}{1.39533}-\frac{\mathrm{i}}{1.39533}+1\right)+17.97966(15.67968)^{\mathrm{k}} \\
& \mathrm{C}_{2} \sum_{\mathrm{i}=0}^{\mathrm{k}}\left(\frac{0.13445}{15.67968}\right)^{\mathrm{i}}\left(\begin{array}{l}
\mathrm{k} \\
\mathrm{i}
\end{array}\right) \times \beta(6+(18.97966) \\
& \left.-\frac{\mathrm{k}}{1.39533}+\frac{\mathrm{i}}{1.39533}-1, \frac{\mathrm{k}}{1.39533}-\frac{\mathrm{i}}{1.39533}+1\right) \\
& \text { From }(10):
\end{aligned}
$$

$$
\begin{aligned}
& \mathrm{C}_{1}=\frac{2}{1}=2 \\
& \mathrm{C}_{2}=\frac{2}{2}=1 \\
& \therefore \mu_{1: 3}^{(\mathrm{k})}[1]=6(15.67968)^{\mathrm{k}} \sum_{\mathrm{i}=0}^{\mathrm{k}}\left(\frac{0.13445}{15.67968}\right)^{\mathrm{i}}\left(\begin{array}{l}
\mathrm{k} \\
\mathrm{i}
\end{array}\right) \\
& \times \beta\left(6+(18.97966)-\frac{\mathrm{k}}{1.39533}+\frac{\mathrm{i}}{1.39533}\right. \\
& \left.-1, \frac{\mathrm{k}}{1.39533}-\frac{\mathrm{i}}{1.39533}+1\right)+17.97966(15.67968)^{\mathrm{k}} \\
& \sum_{i=0}^{k}\left(\frac{0.13445}{15.67968}\right)^{i}\left(\begin{array}{l}
k \\
i
\end{array}\right) \times \beta(6+(18.97966) \\
& \left.-\frac{\mathrm{k}}{1.39533}+\frac{\mathrm{i}}{1.39533}-1, \frac{\mathrm{k}}{1.39533}-\frac{\mathrm{i}}{1.39533}+1\right) \\
& \text { If } \mathrm{k}=1 \text { : } \\
& \therefore \mu_{1: 3}[1]=6(15.67968) \sum_{i=0}^{1}\left(\frac{0.13445}{15.67968}\right)^{i}\left(\begin{array}{l}
1 \\
i
\end{array}\right) \\
& \times \beta\left(6+(18.97966)-\frac{1}{1.39533}+\frac{\mathrm{i}}{1.39533}\right. \\
& \left.-1, \frac{1}{1.39533}-\frac{\mathrm{i}}{1.39533}+1\right)+17.97966(15.67968)
\end{aligned}
$$


Jamjoom, A.A. and Z.A. Al-Saiary /Journal of Mathematics and Statistics 9 (1): 51-61, 2013

Table 2. The expected values and the variances in the presence of multiple outlier from Burr XII with four parameters when $\mathrm{k}=1, \mathrm{n}$ $=5, \mathrm{c}=1.39533, \rho=3, \mathrm{a}=0.13445, \mathrm{~b}=15.67968, \tau=17.97966$

\begin{tabular}{rrrrrrrrrrrr}
\hline $\mathrm{p}$ & $\mathrm{r}$ & \multicolumn{1}{c}{$\mu_{\mathrm{r}: 5}[\mathrm{p}]$} & Variane & $\mathrm{p}$ & $\mathrm{r}$ & \multicolumn{1}{c}{$\mu_{\mathrm{r}: 5}[\mathrm{p}]$} & \multicolumn{1}{c}{ Variane } & $\mathrm{p}$ & $\mathrm{r}$ & $\mu_{\mathrm{r}: 5}[\mathrm{p}]$ & Variane \\
\hline 0 & 1 & 2.27595 & 2.68312 & 1 & 1 & 1.41064 & 0.903247 & 2 & 1 & 1.08231 & 0.489762 \\
0 & 2 & 4.27072 & 5.49707 & 1 & 2 & 3.08853 & 3.342250 & 2 & 2 & 2.09302 & 1.152230 \\
0 & 3 & 6.68235 & 10.71170 & 1 & 3 & 5.37118 & 8.431080 & 2 & 3 & 3.94371 & 5.087640 \\
0 & 4 & 10.26340 & 25.48960 & 1 & 4 & 8.86696 & 22.336100 & 2 & 4 & 7.20476 & 17.890400 \\
0 & 5 & 18.47290 & 146.95700 & 1 & 5 & 16.83670 & 133.319000 & 2 & 5 & 14.85890 & 117.198000 \\
\hline
\end{tabular}

$$
\begin{aligned}
& \sum_{i=0}^{1}\left(\frac{0.13445}{15.67968}\right)^{\mathrm{i}}\left(\begin{array}{l}
1 \\
\mathrm{i}
\end{array}\right) \times \beta\left(6+(18.97966)-\frac{1}{1.39533}\right. \\
& \left.+\frac{\mathrm{i}}{1.39533}-1, \frac{1}{1.39533}-\frac{\mathrm{i}}{1.39533}+1\right)=1.64008
\end{aligned}
$$

More results can be seen in Table 2 for $\mathrm{n}=5, \mathrm{r}=$ $1,2, \ldots, 5$ and $\mathrm{p}=0,1,2$.

Table 1 given below displays the values of the single moments of order statistics in (2.9) when $\mathrm{k}=1 ; \mathrm{n}$ $=5 ; \tau=17.97966 ; \alpha=0.13445 ; \mathrm{b}=15.67968$ and $\mathrm{c}=$ 1.39533 .

\section{Example (2)}

Let $\mathrm{X}_{1}, \mathrm{X}_{2} \sim \mathrm{Barr} \mathrm{XII}$ with $\mathrm{c}=4.873717, \rho=5$ and $X_{3} \sim \operatorname{Normal}(0.644717,0.16199)$. Find $\mu_{1: 3}$

\section{Solution}

A single outlier in this sample that $X_{3} \sim$ Normal (0.644717, 0.16199). But we know that Burr (1942) approximate the normal distribution with Burr XII with two parameters as $c=4.873717$ and $\rho=6.157568$ from which the more accurate values of $\mu, \sigma, \alpha_{3}$ and $\alpha_{4}$ can be obtained as $\mu=0.644717, \sigma=0.161990, \alpha_{3}=0.00000$, $\alpha_{4}=0.00000$. So when $X_{3} \sim$ Normal $(\mu=0.644717, \sigma=$ $0.16199) \Rightarrow X_{3} \sim$ Burr $(c=4.873717), \tau=6.157568$.

Now using Equation 20:

$$
\begin{aligned}
& \therefore \mu_{1: 3}^{(\mathrm{k})}[1]=5 \sum_{\mathrm{s}=\max (0,-1)}^{\min (1,0)} \mathrm{C}_{1} \sum_{\mathrm{l}=0}^{\mathrm{s}}\left(\begin{array}{l}
\mathrm{s} \\
1
\end{array}\right) \sum_{\mathrm{m}=0}^{\mathrm{r}-\mathrm{s}-1} \\
& \left(\begin{array}{c}
\mathrm{r}-\mathrm{s}-1 \\
\mathrm{~m}
\end{array}\right)(-1)^{1+\mathrm{m}} \beta\left(\Phi-\frac{\mathrm{k}}{4.873717}-1, \frac{\mathrm{k}}{4.873717}+1\right) \\
& +6.157568 \sum_{\mathrm{s}=\max (0,0)}^{\min (2,0)} \mathrm{C}_{2} \sum_{1=0}^{\mathrm{s}}\left(\begin{array}{c}
\mathrm{s} \\
1
\end{array}\right) \sum_{\mathrm{m}=0}^{\mathrm{r}-\mathrm{s}-1}\left(\begin{array}{c}
\mathrm{r}-\mathrm{s}-1 \\
\mathrm{~m}
\end{array}\right)(-1)^{1+\mathrm{m}} \\
& \beta\left(\Phi-\frac{\mathrm{k}}{4.873717}-1, \frac{\mathrm{k}}{4.873717}+1\right) \\
& \Phi=5(3-1-\mathrm{s}+1)+(6.157568)(1-1+\mathrm{s}+1+\mathrm{m}) \\
& +1=5(2-\mathrm{s})+(6.157568)(\mathrm{s}+\mathrm{m}+1)
\end{aligned}
$$

$$
\begin{aligned}
& \therefore \mu_{1: 3}^{(\mathrm{k})}[1]=5 \sum_{\mathrm{s}=0}^{0} \mathrm{C}_{1} \sum_{\mathrm{l}=0}^{\mathrm{s}}\left(\begin{array}{l}
\mathrm{s} \\
1
\end{array}\right) \sum_{\mathrm{m}=0}^{1-\mathrm{s}-1}\left(\begin{array}{c}
1-\mathrm{s}-1 \\
\mathrm{~m}
\end{array}\right) \\
& (-1)^{1+\mathrm{m}} \beta\left(\Phi-\frac{\mathrm{k}}{4.873717}-1, \frac{\mathrm{k}}{4.873717}+1\right) \\
& +6.157568 \sum_{s=0}^{0} C_{2} \sum_{1=0}^{s}\left(\begin{array}{l}
s \\
1
\end{array}\right) \sum_{m=0}^{1-s-1}\left(\begin{array}{c}
1-s-1 \\
m
\end{array}\right) \\
& (-1)^{1+\mathrm{m}} \beta\left(\Phi-\frac{\mathrm{k}}{4.873717}-1, \frac{\mathrm{k}}{4.873717}+1\right) \\
& \therefore \mu_{1: 3}^{(\mathrm{k})}[1]=5 \mathrm{C}_{1}(1) \beta \\
& \left(\begin{array}{l}
5(2)+6.157568(1)+1 \\
-\frac{\mathrm{k}}{4.873717}-1, \frac{\mathrm{k}}{4.873717}+1
\end{array}\right) \\
& +6.157568 \mathrm{C}_{2}(1) \beta \\
& \left(\begin{array}{l}
5(2)+6.157568(1)+ \\
1-\frac{\mathrm{k}}{4.873717}-1, \frac{\mathrm{k}}{4.873717}+1
\end{array}\right)
\end{aligned}
$$

From (10):

$\mathrm{C}_{1}=\frac{2}{1}=2$

$\mathrm{C}_{2}=\frac{2}{2}=1$

$\therefore \mu_{1: 3}^{(\mathrm{k})}[1]=10 \times \beta$

$$
\begin{aligned}
& \left(\begin{array}{l}
5(2)+6.157568(1)+ \\
1-\frac{\mathrm{k}}{4.873717}-1, \frac{\mathrm{k}}{4.873717}+1
\end{array}\right) \\
& +6.157568 \times \beta \\
& \left(\begin{array}{l}
5(2)+6.157568(1)+1 \\
-\frac{\mathrm{k}}{4.873717}-1, \frac{\mathrm{k}}{4.873717}+1
\end{array}\right)
\end{aligned}
$$

If $\mathrm{k}=1$ :

$$
\begin{aligned}
& \therefore \mu_{1: 3}^{(\mathrm{k})}[1]=(10+6.157568) \\
& \beta\left(17.157568-\frac{1}{4.873717}-1, \frac{1}{4.873717}+1\right) \\
& \quad=0.522053
\end{aligned}
$$


Table 3. The expected values and the variances in the presence of multiple outlier from Burr XII with two parameters when $\mathrm{k}=1, \mathrm{n}$

\begin{tabular}{llllllllllll}
\multicolumn{10}{c}{$=5, \mathrm{c}=4.873717$ and $\rho=3, \tau=6.157568$} \\
\hline $\mathrm{p}$ & $\mathrm{r}$ & $\mu_{\mathrm{r}: 3}[\mathrm{p}]$ & Variane & $\mathrm{p}$ & $\mathrm{r}$ & $\mu_{\mathrm{r}: 3}[\mathrm{p}]$ & Variane & $\mathrm{p}$ & $\mathrm{r}$ & $\mu_{\mathrm{r}: 3}[\mathrm{p}]$ & Variane \\
\hline 0 & 1 & 0.530399 & 0.0163404 & 1 & 1 & 0.509259 & 0.0149178 & 2 & 1 & 0.492270 & 0.01384444 \\
0 & 2 & 0.660577 & 0.0122812 & 1 & 2 & 0.636263 & 0.0112900 & 2 & 2 & 0.614333 & 0.01031460 \\
0 & 3 & 0.759818 & 0.0139870 & 1 & 3 & 0.734454 & 0.0110687 & 2 & 3 & 0.709143 & 0.01006590 \\
0 & 4 & 0.862285 & 0.0139870 & 1 & 4 & 0.836981 & 0.0134756 & 2 & 4 & 0.809389 & 0.01249790 \\
0 & 5 & 1.012800 & 0.0260662 & 1 & 5 & 0.988464 & 0.0257907 & 2 & 5 & 0.959827 & 0.02496020 \\
\hline
\end{tabular}

More results can be seen in Table 3 for $n=5, r=$ $1,2, \ldots, 5$ and $\mathrm{p}=0,1,2$.

\subsection{Moments of Order Statistics from Burr XII with Four Parameters Using Theorem of Barakat and Abdelkader (2004)}

Let $\mathrm{X}_{1}, \mathrm{X}_{2}, \ldots, \mathrm{X}_{\mathrm{n}}$ be independent nonidentically distributed r.v's. The $\mathrm{k}^{\text {th }}$ moment of all order statistics, $\mu_{\mathrm{r}: \mathrm{n}}^{(\mathrm{k})}$ for $1 \leq \mathrm{r} \leq \mathrm{n}$ and $\mathrm{k}=1,2, \ldots$ is given by (Barakat and Abdelkader, 2004) Equation 23:

$\mu_{\mathrm{r}: \mathrm{n}}^{(\mathrm{k})}=\sum_{\mathrm{j}=\mathrm{n}-\mathrm{r}+1}^{\mathrm{n}}(-1)^{\mathrm{j}-(\mathrm{n}-\mathrm{r}+\mathrm{l})}\left(\begin{array}{c}\mathrm{j}-1 \\ \mathrm{n}-\mathrm{r}\end{array}\right) \mathrm{I}_{\mathrm{j}}(\mathrm{k})$

Where Equation 24:

$$
\begin{aligned}
& I_{j}(k)=\sum_{1 \leq i_{1}<i_{2}<\cdots<i_{j} \leq n} \ldots \\
& \sum k \int_{0}^{\infty} x^{k-1} \prod_{t=1}^{j} G_{i_{t}}(x) d x, j=1,2, \cdots, n
\end{aligned}
$$

$\mathrm{G}_{\mathrm{i}}(\mathrm{x})=1-\mathrm{F}_{\mathrm{i}}(\mathrm{x})$, with $\left(\mathrm{i}_{1}, \mathrm{I}_{2}, \ldots, \mathrm{i}_{\mathrm{n}}\right)$ is a permutation of $(1,2, \ldots, n)$ for which $i_{1} \leq i_{2}<\ldots<i_{n}$.

We consider the case when the variable $X_{1}, X_{2}, \ldots$, $X_{n-p}$ are independent observations from Burr XII with four parameters distribution with density Equation 25:

$$
\begin{aligned}
& f(x)=\frac{\rho c}{b}\left(1+\left(\frac{x-a}{b}\right)^{c}\right)^{-\rho-1} \\
& \left(\frac{x-a}{b}\right)^{c-1}, \\
& a \leq x \leq \infty, \rho>0, c>0, b>0
\end{aligned}
$$

The corresponding cumulative distribution function $\mathrm{F}(\mathrm{x})$ is given as:

$$
\begin{aligned}
& F(x)=1-\left(1+\left(\frac{x-a}{b}\right)^{c}\right)^{-\rho}, \\
& x \geq a, \rho>0, c>0, b>0
\end{aligned}
$$

Using (4) in (2):

$$
\begin{aligned}
& \mathrm{I}_{\mathrm{j}}(\mathrm{k})=\sum_{1 \leq \mathrm{i}_{1}<\mathrm{i}_{2}<\cdots<\mathrm{i}_{\mathrm{j}} \leq \mathrm{n}} \cdots \\
& \sum k \int_{a}^{\infty} x^{k-1}\left(1+\left(\frac{x-a}{b}\right)^{c}\right)^{-\sum_{t=1}^{j} \rho_{i_{t}}} d x \\
& \text { Substituting } y=\frac{x-a}{b} \text { : } \\
& \Rightarrow \mathrm{x}=\mathrm{by}+\mathrm{a} \Rightarrow \mathrm{dx}=\mathrm{bdy} \\
& \text { at } x=a \Rightarrow y=0 \text {, at } x=\infty \Rightarrow y=\infty
\end{aligned}
$$

The above Equation reduces to:

$$
\begin{aligned}
& \mathrm{I}_{\mathrm{j}}(\mathrm{k})=\mathrm{k} \sum_{1 \leq \mathrm{i}_{1}<\mathrm{i}_{2}<\cdots<\mathrm{i}_{\mathrm{j}} \leq \mathrm{n}} \cdots \\
& \sum b \int_{0}^{\infty}(b y+a)^{k-1}\left(1+y^{c}\right)^{-\sum_{t=1}^{j} \rho_{i_{t}}} d y \\
& =\mathrm{k} \sum_{1 \leq \mathrm{i}_{1}<\mathrm{i}_{2}<\cdots<\mathrm{i}_{\mathrm{j}} \leq \mathrm{n}} \ldots \\
& \left.\sum \mathrm{b} \int_{0}^{\infty} \sum_{\mathrm{t}=0}^{\mathrm{k}-1} \sum_{\mathrm{t}=1}^{\mathrm{j}} \rho_{\mathrm{i}_{\mathrm{t}}}^{\mathrm{k}-1} \mathrm{dy}_{\mathrm{m}}\right) \mathrm{a}^{\mathrm{m}}(\mathrm{b} \mathrm{y})^{\mathrm{k}-\mathrm{m}-1}\left(1+\mathrm{y}^{\mathrm{c}}\right) \\
& \mathrm{k} \sum_{1 \leq \mathrm{i}_{1}<\mathrm{i}_{2}<\cdots<\mathrm{i}_{\mathrm{j}} \leq \mathrm{n}} \cdots \\
& \sum \sum_{m=0}^{k-1} b^{k-m}\left(\begin{array}{c}
k-1 \\
m
\end{array}\right) a^{m} \int_{0}^{\infty}(y)^{k-m-1}-\sum_{t=1}^{j} \rho_{i_{t}} d y
\end{aligned}
$$

Upon using:

$$
\int_{0}^{\infty}(y)^{k-1}\left(1+y^{c}\right)^{-\alpha} d y=\frac{1}{c} \beta\left(\alpha-\frac{k}{c}, \frac{k}{c}\right)
$$

where, $\beta(a, b)$ is the regular beta function: 
Jamjoom, A.A. and Z.A. Al-Saiary /Journal of Mathematics and Statistics 9 (1): 51-61, 2013

Table 4. The expected values and the variances in the presence of multiple outlier using Barakat and Abdelkader (2004) and method from Burr XII with four parameters when $\mathrm{k}=1, \mathrm{n}=5, \mathrm{c}=1.39533, \rho=3, \mathrm{a}=0.13445, \mathrm{~b}=15.67968, \tau=17.97966$

\begin{tabular}{|c|c|c|c|c|c|c|c|c|c|c|c|}
\hline $\mathrm{p}$ & $\mathrm{r}$ & $\mu_{\mathrm{r}: 3}[\mathrm{p}]$ & Variane & $\mathrm{p}$ & $\mathrm{r}$ & $\mu_{\mathrm{r}: 3}[\mathrm{p}]$ & Variane & $\mathrm{p}$ & $\mathrm{r}$ & $\mu_{\mathrm{r}: 3}[\mathrm{p}]$ & Variane \\
\hline$\overline{0}$ & 1 & 2.14150 & 3.25897 & 1 & 1 & 1.27619 & 1.24641 & 2 & 1 & 0.947864 & 0.744643 \\
\hline 0 & 2 & 4.13627 & 6.60931 & 1 & 2 & 2.95408 & 4.13660 & 2 & 2 & 1.958570 & 1.678890 \\
\hline 0 & 3 & 6.54790 & 12.47240 & 1 & 3 & 5.23673 & 9.83924 & 2 & 3 & 3.809260 & 6.111950 \\
\hline 0 & 4 & 10.12900 & 28.21320 & 1 & 4 & 8.73251 & 24.68430 & 2 & 4 & 7.070310 & 19.791600 \\
\hline 0 & 5 & 18.33840 & 151.88900 & 1 & 5 & 16.70230 & 137.81000 & 2 & 5 & 14.724500 & 121.158000 \\
\hline
\end{tabular}

$$
\begin{aligned}
& \mathrm{I}_{\mathrm{j}}(\mathrm{k})=\mathrm{k} \sum_{1 \leq \mathrm{i}_{1}<\mathrm{i}_{2}<\cdots<\mathrm{i}_{\mathrm{j}} \leq \mathrm{n}} \cdots \\
& \sum \sum_{\mathrm{m}=0}^{\mathrm{k}-1} \frac{\mathrm{b}^{\mathrm{k}-\mathrm{m}}}{\mathrm{c}}\left(\begin{array}{c}
\mathrm{k}-1 \\
\mathrm{~m}
\end{array}\right) \mathrm{a}^{\mathrm{m}} \beta\left(\sum_{\mathrm{t}=1}^{\mathrm{j}} \rho_{\mathrm{i}_{\mathrm{t}}}-\frac{\mathrm{k}-\mathrm{m}}{\mathrm{c}}, \frac{\mathrm{k}-\mathrm{m}}{\mathrm{c}}\right) \\
& \mu_{\mathrm{r}: \mathrm{n}}^{(\mathrm{k})}=\sum_{\mathrm{j}=\mathrm{n}-\mathrm{r}+1}^{\mathrm{n}}(-1)^{\mathrm{j}-(\mathrm{n}-\mathrm{r}+1)}\left(\begin{array}{c}
\mathrm{j}-1 \\
\mathrm{n}-\mathrm{r}
\end{array}\right) \\
& \times \mathrm{k} \sum_{1 \leq \mathrm{i}_{1}<\mathrm{i}_{2}<\cdots<\mathrm{i}_{\mathrm{j}} \leq \mathrm{n}} \cdots \\
& \sum \sum_{\mathrm{m}=0}^{\mathrm{k}-1} \frac{\mathrm{b}^{\mathrm{k}-\mathrm{m}}}{\mathrm{c}}\left(\begin{array}{c}
\mathrm{k}-1 \\
\mathrm{~m}
\end{array}\right) \mathrm{a}^{\mathrm{m}} \beta\left(\sum_{\mathrm{t}=1}^{\mathrm{j}} \rho_{\mathrm{i}_{\mathrm{t}}}-\frac{\mathrm{k}-\mathrm{m}}{\mathrm{c}}, \frac{\mathrm{k}-\mathrm{m}}{\mathrm{c}}\right) \\
& \text { If } \mathrm{k}=1 \text { : } \\
& \mu_{r: n}=\sum_{j=n-r+1}^{n}(-1)^{j-(n-r+1)}\left(\begin{array}{c}
j-1 \\
n-r
\end{array}\right) \\
& \times \sum_{1 \leq \mathrm{i}_{1}<\mathrm{i}_{2}<\cdots<\mathrm{i}_{\mathrm{j}} \leq \mathrm{n}} \cdots \sum \frac{\mathrm{b}}{\mathrm{c}} \beta\left(\sum_{\mathrm{t}=1}^{\mathrm{j}} \rho_{\mathrm{i}_{\mathrm{t}}}-\frac{1}{\mathrm{c}}, \frac{1}{\mathrm{c}}\right)
\end{aligned}
$$

\section{Example}

Let $\mathrm{X}_{1}, \mathrm{X}_{2} \sim$ Barr XII with $\mathrm{c}=1.39533, \rho=3, \mathrm{a}=$ $0.13445, b=15.67968$ and $X_{3} \sim \operatorname{Gamma}(\beta=1, \alpha=$ 2). Find $\mu_{1: 3}$.

\section{Solution}

$\mathrm{X}_{3} \sim \operatorname{Gamma}(\beta=1, \alpha=2) \Rightarrow \mathrm{X}_{3} \sim$ Burr IIX with parameters $(c=1.39533, \rho=17.97966, a=0.13445, b=$ 15.67968):

$$
\begin{gathered}
\mu_{1: 3}=\sum_{\mathrm{j}=3}^{3}(-1)^{\mathrm{j}-3}\left(\begin{array}{c}
\mathrm{j}-1 \\
2
\end{array}\right) \\
\sum_{1 \leq \mathrm{i}_{1}<\mathrm{i}_{2}<\cdots<\mathrm{i}_{\mathrm{j}} \leq 3} \ldots \\
\sum_{\mathrm{c}}^{\mathrm{b}} \beta\left(\sum_{\mathrm{t}=1}^{\mathrm{j}} \rho_{\mathrm{i}_{\mathrm{t}}}-\frac{1}{\mathrm{c}}, \frac{1}{\mathrm{c}}\right) \\
=\sum_{1 \leq \mathrm{i}_{1}<\mathrm{i}_{2}<\cdots<\mathrm{i}_{3} \leq 3} \ldots
\end{gathered}
$$

$$
\begin{aligned}
& \sum \frac{\mathrm{b}}{\mathrm{c}} \beta\left(\sum_{\mathrm{t}=1}^{3} \rho_{\mathrm{i}_{\mathrm{t}}}-\frac{1}{\mathrm{c}}, \frac{1}{\mathrm{c}}\right) \\
& \quad=\frac{\mathrm{b}}{\mathrm{c}} \beta\left(\rho_{1}+\rho_{2}+\rho_{3}-\frac{1}{\mathrm{c}}, \frac{1}{\mathrm{c}}\right) \\
& =\frac{15.67968}{1.39533} \beta(3+3+17.97966 \\
& \left.-\frac{1}{1.39533}, \frac{1}{1.39533}\right) \\
& =1.50563
\end{aligned}
$$

More results can be seen in Table 4 for $\mathrm{r}=$ $1,2, \ldots, 5$ and $\mathrm{p}=0,1,2$.

\section{CONCLUSION}

We can find moments of order statistics from independent and nonidentically distributed random variables for any distribution with no simple closed from using approximating idea with Burr XII or any other distribution.

\section{REFRENCES}

Arnold, B.C. and N. Balakrishnan, 1989. Relations, Bounds and Approximations for Order Statistics. 1st Edn., Springer-Verglas, New York, ISBN-10: 0387969756, pp: 173.

Balakrishnan, N. and K. Balasubramanian, 1995. Order statistics from non-identical power function random variables. Commun. Stat. Theor. Meth., 24: 14431454. DOI: 10.1080/03610929508831564

Balakrishnan, N., 1994. Order statistics from nonidentical exponential random variables and some applications. Comput. Stat. Data Anal., 18: 203-238. DOI: 10.1016/0167-9473(94)90172-4

Barakat, H. and Y. Abdelkader, 2004. Computing the moments of order statistics from nonidentical random variables. Stat. Meth. Appl., 13: 15-26. DOI: $10.1007 / \mathrm{s} 10260-003-0068-9$ 
Barnett, V. and T. Lewis, 1994. Outliers in Statistical Data. 3rd Edn., Wiley and Sons, Chichester, ISBN10: 0471930946, pp: 584.

Burr, I.W., 1942. Cumulative frequency functions. Annals Math. Stat., 13: 215-232.

Burr, I.W., 1967. A useful approximation to the normal distribution function, with application to simulation. Technometrics, 9: 647-651. DOI: 10.1080/00401706.1967.10490512

Burr, I.W., 1973. Parameters for a general system of distributions to match a grid of á3 and á4. Commun. Stat, 2: 1-21.

Childs, A, 1996. Advances in statistical inference and outlier related issues. Ph.D. Thesis, McMaster University.

Childs, A., N. Balakrishnan and M. Moshref, 2001. Order statistics from non-identical right-truncated Lomax random variables with applications. Stat. Papers, 42: 187-206.

Hartley, H.O. and H.A. David, 1978. Contributions to Survey Sampling and Applied Statistics. 1st Edn., Academic Press, San Francisco, ISBN-10: 0122047508, pp: 318.
Launer, C.A. and T.D. Bills, 1979. Influences of Selected Environmental Factors on the Activity of a Prospective Fish Toxicant, 2-(Digeranylamino)Ethanol, in Laboratory Tests. 1st Edn., Department of the Interior, Washington, pp: 4.

Moshref, M.E. and K.S. Sultan, 2007. Moments of order statistics from Rayleigh distribution in the presence of outlier observations. Department of Mathematics, Al-Azhar University,

Shu, V.S., 1978. Robust Estimation of a Location Parameter in the Presence of Outliers. 1st Edn., Iowa, Amis, pp: 372.

Tadikamalla, P.R. and J.S. Ramberg, 1975. An approximate method for generating gamma and other variables. J. Stat. Comput. Simul., 3: 375-382.

Tadikamalla, P.R., 1977. An approximation to the moments and the percentiles of gamma order statistics. Sankhya: IJS, 39: 372-381.

Vaughan, R.J. and W.N. Venables, 1972. Permanent expressions for order statistic densities. J. Royal Stat. Soc., 34: 308-310.

Wheeler, D.J., 1975. An approximation for simuiation of gamma distributions. J. Stat. Comput. Simul., 3: 225-232. DOI: 10.1080/00949657508810088 